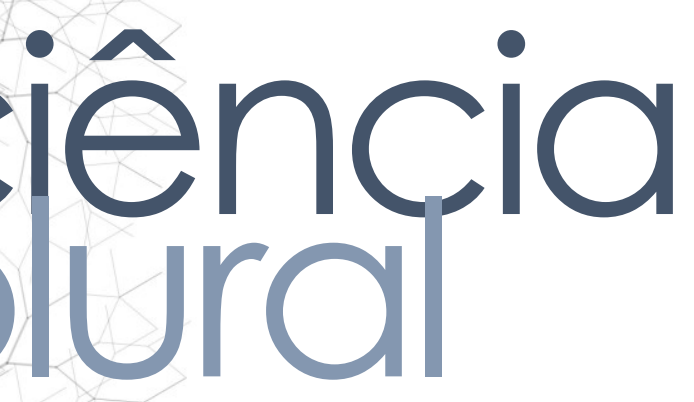

\title{
ESTRATÉGIAS PARA PREVISÃO DA DEMANDA POR SERVIÇOS ODONTOLÓGICOS: UMA REVISÃO INTEGRATIVA
}

Strategies for predicting demand for dental services: an integrative review

Estrategias para pronosticar la demanda de servicios dentales: una revisión integradora

Lorena Tatim Farhat • Pontifícia Universidade Católica do Paraná/ PUC-PR • Mestranda do Programa de Pós-Graduação em Tecnologia em Saúde da PUC-PR • E-mail: lorenatatim@hotmail.com

Ana Luísa Gomes Coelho • Mestranda do Programa de Pós-Graduação em Tecnologia em Saúde da PUC-PR • Mestre em Gestão de Saúde pela Università Cattolica del Sacro Cuore $\bullet$ Cirurgiã-Dentista pela Universidade Positivo e Administradora pela FAE Business School. • E-mail: analuisagcoelho@gmail.com

Deborah Ribeiro Carvalho • Professora do Programa de Pós-Graduação em Tecnologia em Saúde da PUC-PR • Doutora em Computação de Alto Desempenho pela Universidade Federal do Rio Janeiro (COPPE)•E-mail: drdrcarvalho@gmail.com

Autora responsável pela correspondência:

Lorena Tatim Farhat・ E-mail: lorenatatim@hotmail.com 


\section{RESUMO}

Introdução: A previsão da demanda por serviços de saúde possibilita melhorias no processo de tomada de decisões gerenciais nas organizações de saúde. Objetivo: $O$ estudo buscou identificar, na literatura, as metodologias utilizadas para prever a demanda por serviços odontológicos. Metodologia: Trata-se de uma revisão integrativa conduzida de acordo com o método PRISMA. A expressão de busca (((forecast* OR "prediction" OR "prevision") AND ("demand" OR trend*) AND ("dental services"))) foi aplicada em campos determinados para os repositórios Scopus Elsevier, Pubmed, BVS e IEEE Xplore. As metodologias empregadas foram categorizadas conforme a temporalidade e o instrumento empregado nos estudos. Resultados: Após aplicação do processo de seleção-exclusão baseado na recomendação PRISMA, foram selecionados 34 documentos, classificados conforme a dependência da colaboração do paciente, a procedência de auto avaliações, o direcionamento a um grupo populacional ou demanda específica, e a relação com o setor público, privado ou ambos. As metodologias identificadas foram: estudos de temporalidade transversal em que o instrumento foi a aplicação de questionário; estudos de temporalidade transversal nos quais o instrumento foi a observação; e estudo de temporalidade longitudinal onde o instrumento foi a aplicação de questionário. Conclusões: A partir dos resultados dessa revisão, foi gerado conhecimento capaz de apoiar o delineamento de estudos com potencial para aprimorar a previsibilidade da demanda por serviços odontológicos.

Palavras-Chave: Necessidades e Demandas de Serviços de Saúde; Assistência à Saúde; Serviços de Saúde Bucal; Apoio ao Planejamento em Saúde.

\section{ABSTRACT}

Introduction: Forecasting the demand for health services enables improvements in the decision-making process in healthcare organizations. Objective: The present study aimed to identify in the literature the methodologies used to predict the demand for dental services. Methodology: This was an integrative review conducted in accordance with the PRISMA method. The search expression (((forecast* OR "prediction" OR "prevision") AND (“demand" OR trend*) AND (“dental services"))) was applied to fields determined for the Scopus Elsevier, PubMed, VHL, and IEEE Xplore databases. The identified methodologies were categorized according to temporality and instrument used in the studies. Results: After applying the selectionexclusion process based on Prisma, 34 documents were selected, classified according to patient's collaboration dependence, self-assessments, the targeting of a specific group or demand and the relationship with public, private or both sectors. The methodologies identified were transversal temporality in which the instrument was e application of a questionnaire or observation and longitudinal temporality where e instrument was the application of a questionnaire. Conclusions: From the results this review, insights were generated capable of supporting studies with potential to prove the forecast of dental services. 
Keywords: Health Services Needs and Demand; Delivery of Health Care; Dental Health Services; Health Planning Support.

\section{RESUMEN}

Introducción: La predicción de la demanda de servicios de salud permite mejorar el proceso de decisiones em organizaciones de salud. Objetivo: El estudio buscó identificar, en la literatura, las estrategias utilizadas para predecir la demanda de servicios dentales. Metodología: Esta es una revisión integradora realizada de acuerdo con el método PRISMA. La expresión de búsqueda (((pronóstico * O "predicción" O "previsión") Y ("demanda" O tendencia *) Y ("servicios dentales"))) se aplicó a los campos determinados para repositorios Scopus Elsevier, Pubmed, BVS y IEEE Xplore. Las metodologías identificadas se categorizaron según la temporalidad y el instrumento utilizado em los estudios. Resultados: Tras aplicar el proceso de selección-exclusión basado em Prisma, se seleccionaron 34 documentos, clasificados según la dependencia de colaboración del paciente, las autoevaluaciones a, la focalización de un grupo o demanda específica y la relación con el sector público, privado o ambos. Las metodologías identificadas fueron: la temporalidad transversal en los cuales el instrumento era la aplicación de un cuestionario; estudios de temporalidad transversal en los cuales la observación era el instrumento; y estudio de temporalidad longitudinal donde el instrumento fue la aplicación de un cuestionario. Conclusiones: A partir de los resultados de esta revisión se generó conocimiento capaz de sustentar el diseño de estudios con potencial para mejorar la predictibilidad de la demanda de servicios odontológicos.

Palabras clave: Necesidades y demandas de servicios de salud; Prestación de Atención de Salud; Servicios de Salud Dental; Apoyo a la planificación de la salud. 


\section{Introdução}

As previsões da demanda em saúde requerem tanto informações socioeconômicas e do estado de saúde da população, como do perfil de utilização de serviços e equipamentos ${ }^{1}$. Para os serviços odontológicos tanto as informações epidemiológicas quanto o poder de compra da população são determinantes².

Entretanto, é consenso que o estado de saúde é mais determinante do que os fatores socioeconômicos para estimar a utilização dos serviços de saúde ${ }^{1}$. Por isso, as informações epidemiológicas são usadas para avaliar a extensão e a severidade das doenças odontológicas das populações e, a partir dessas avaliações, torna-se possível a condução de estimativas sobre a necessidade dos serviços ${ }^{3}$.

Estudos sobre a utilização desses serviços permitem caracterizar a população assistida, identificar suas condições de saúde e a motivação por sua procura, sendo aspectos fundamentais para melhorar a oferta e organizar as ações de saúde ${ }^{4}$. Nesse contexto, as condições macroeconômicas podem afetar diretamente o acesso a cuidados odontológicos por impactarem no financiamento de tratamentos de saúde que podem afetar a disponibilidade dos serviços odontológicos públicos e a extensão do subsídio para tais serviços ${ }^{5}$.

A previsão da demanda por serviços de saúde é um fator importante para a tomada de decisões gerenciais de todas as organizações de saúde ${ }^{6}$. Porém, essas projeções foram reconhecidas ineficientes, uma vez que as particularidades que envolvem a assistência em saúde impedem o comportamento independente da demanda7.

Somam-se, ainda, o emprego de materiais e equipamentos, as expertises e preferências do profissional de saúde na definição do tratamento efetivado ${ }^{8}$. Assim, nos serviços odontológicos, a força de trabalho está diretamente ligada à demanda, juntamente ao tamanho e às características da população, aos cenários econômico e de oferta de serviços público e privado e às crenças de saúde da população 9 .

Estudos conduzidos no exterior têm demonstrado diminuição na utilização de viços odontológicos ${ }^{10-12}$. No Brasil, foi registrado um aumento de $137 \%$ nos cursos Odontologia entre os anos de 1991 e 200813. Além disso, houve crescimento de \% no mercado de planos odontológicos entre 2010 e $2016^{14}$ e de 7,2\% no período 
de dezembro de 2018 a dezembro de 201915. Situação oposta foi observada em países desenvolvidos, que registram uma relação entre oferta e demanda por serviços odontológicos distante da brasileira ${ }^{16,17}$.

Diante do exposto, o objetivo do presente estudo foi identificar as metodologias utilizadas para previsão de demanda por serviços odontológicos, destacando aquelas mais utilizadas. A partir dos resultados desta revisão espera-se gerar conhecimento acerca dessas metodologias com potencial para propiciar melhorias na gestão das organizações de saúde.

\section{Metodologia}

Trata-se de uma revisão integrativa da literatura, por permitir a compilação do conhecimento científico já produzido e viabilizar a análise do tema investigado e a avaliação da pertinência dos procedimentos empregados ${ }^{18}$. O percurso metodológico da revisão integrativa foi alicerçado na recomendação PRISMA ${ }^{19}$. Assim, o processo de seleção-exclusão consistiu de quatro etapas: 1) identificação - verificação do número de documentos encontrados nas bases de dados selecionadas e leitura dos títulos dos documentos selecionados para eliminação dos textos que não atendiam aos critérios de inclusão; 2) seleção - leitura dos títulos dos documentos encontrados para eliminação daqueles em duplicidade; 3) elegibilidade - leitura dos resumos dos documentos selecionados e eliminação dos estudos que não atendiam aos critérios de inclusão, avaliação dos documentos restantes por meio da leitura completa do texto e nova eliminação daqueles que não atenderam aos critérios de inclusão; 4) inclusão síntese das informações evidenciadas nos artigos selecionados.

Na etapa de inclusão a síntese das informações se deu por meio da extração dos dados dos textos selecionados. Esse processo foi conduzido em acordo com diretrizes da literatura que preconizam dois estágios: 1) avaliação dos dados, a qual inclui terpretação imparcial dos dados sob à luz de uma síntese inovadora da evidência; e redução dos dados, que abrange a determinação de um sistema de classificação que rmita o gerenciamento dos dados provenientes de metodologias diversas ${ }^{20}$. Dessa 
maneira, foi definido que os aspectos relevantes referentes às metodologias adotadas seriam a temporalidade, o instrumento e o modelo de análise de dados empregado.

O critério de inclusão foi abordar no título e/ou no resumo estratégias aplicadas para prever ou avaliar a demanda por serviços odontológicos. Foram excluídos relatos de caso, textos com abstract ausente (notas do autor, prefácio, entre outros) e revisões de literatura. Não houve restrição referente ao período e tipo de publicação e os idiomas dos textos incluídos foram português, inglês e espanhol.

A pesquisa foi realizada no dia 04 de agosto de 2020, via acesso remoto. Como a busca e a análise dos documentos foram conduzidas pelo mesmo autor, não foi adotada etapa de treinamento.

As bases de dados selecionadas foram: Scopus, BVS, IEEE e Pubmed. Adotouse, em todas as bases de dados, a modalidade "busca avançada" com os seguintes descritores e operadores booleanos: (((forecast* OR “prediction" OR "prevision") AND ("demand" OR trend*) AND ("dental services"))). A expressão de busca foi aplicada nos campos título, resumo e descritores. Como somente a base de dados Scopus permite a busca simultânea nos três campos, para os repositórios BVS, IEEE e Pubmed foram conduzidas pesquisas específicas para cada campo. Os mesmos critérios de inclusão e exclusão foram adotados em todas as etapas e os resultados de cada repositório foram registrados.

Dos artigos que atenderam a todos os critérios de seleção, após o processo de seleção-exclusão adotado, foram extraídas as seguintes características: a) autor, ano de publicação e país de origem do estudo; b) objetivo, que se refere à descrição da lacuna científica que o estudo buscou solucionar; c) temporalidade e instrumento empregados na pesquisa; d) modelo de análise dos dados e setor da saúde referido; e) resultados obtidos. Com o objetivo de verificar a reprodutibilidade dos desfechos relatados em cenários diversos, os resultados descritos foram arranjados conforme a dependência da colaboração do paciente, a procedência de auto avaliações, o direcionamento a um rupo populacional específico ou a uma demanda específica, e a relação com o setor úblico, privado ou ambos (híbrido). Esse arranjo procurou, também, possibilitar a scussão de resultados alcançados a partir de metodologias distintas. 


\section{ciêncíncia
puurl}

\section{Resultados}

Foram identificados 297 documentos nos repositórios: Scopus Elsevier (65), Pubmed (175), BVS (57), IEEE Xplore (0). Estudos duplicados foram desconsiderados em acordo com a ordem de busca: Scopus, Pubmed e BVS. A Figura 1 descreve o processo de seleção-exclusão até a obtenção dos 34 documentos incluídos 2, 5, 10, 21-51. As características extraídas dos artigos foram sintetizadas no quadro 1.

Dos documentos selecionados, o estudo mais antigo foi publicado em $1984^{21} \mathrm{e}$ o mais recente em 201851. Os estudos foram conduzidos em diversos países, sendo a sua maioria nos Estados Unidos. Dois estudos brasileiros atenderam aos critérios de seleção e foram incluídos nesta revisão ${ }^{10,35}$.

As metodologias empregadas foram: estudos de temporalidade transversal em que o instrumento foi a aplicação de questionário 5,10, 22-24, 27, 29, 35, 37-40, 42-44, 51; estudos de temporalidade transversal nos quais o instrumento foi a observação ${ }^{2,}$, 21, 28, 30-34, 36, 41, 4548-50; e estudo de temporalidade longitudinal onde o instrumento foi a aplicação de questionário $^{26}$. Destas, a mais prevalente foi o estudo de temporalidade transversal em que o instrumento foi a aplicação de questionário, empregada em 41,93\% dos estudos.

Em se tratando do modelo de análise, o mais usual foi a regressão logística, empregada em mais da metade dos textos $2,5,10,24,26,27,29,31,32-36,38,40,42,43,45,51$, seguida pela distribuição de frequência, utilizada em 20,59\% dos estudos. A regressão linear ${ }^{23}$, 41, a análise discriminante ${ }^{25}$, a regressão de Poison $^{44}$, o geoprocessamento ${ }^{46-48}$ e a análise de correlação ${ }^{50}$ foram os modelos menos recorrentes.

Aproximadamente $65 \%$ dos estudos não dissociou os setores da saúde em público e privado para avaliar a demanda sendo, portanto, classificados como híbridos 2, 5, 21, 22, 23-25, 27-29, 23,35-37, 40-44, 51. Seis autores avaliaram o setor público 26, 34, 38, 46, 48 e cinco o setor privado $2,30-32,39$. 
Figura 1. Processo de seleção-exclusão em acordo com a metodologia PRISMA. Curitiba-PR, 2020

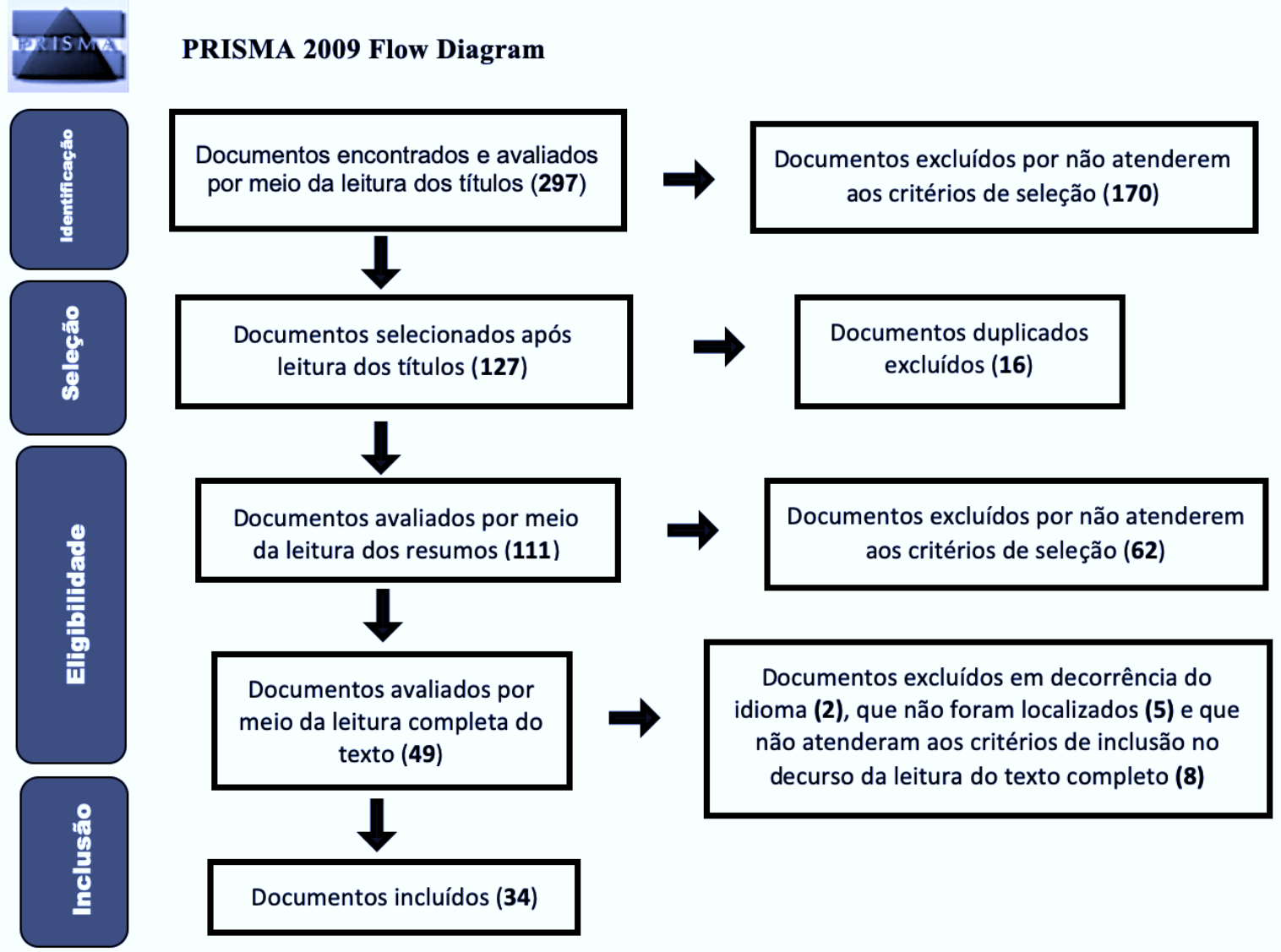

Fonte: os autores - adaptado de MOHER et al. (2009)

Quadro 1. Síntese das características extraídas após leitura completa dos textos, de acordo com o autor, ano de publicação e país de origem do estudo. Curitiba-PR, 2020

\begin{tabular}{|c|c|c|c|}
\hline $\begin{array}{l}\text { AUTOR/ANO DE } \\
\text { PUBLICAÇÃO/ } \\
\text { PAÍS DE ORIGEM } \\
\end{array}$ & $\begin{array}{l}\text { TEMPORALIDADE/ } \\
\text { INSTRUMENTO DO } \\
\text { ESTUDO }\end{array}$ & $\begin{array}{c}\text { MODELO DE } \\
\text { ANÁLISE/ SETOR }\end{array}$ & RESULTADOS ALCANÇADOS \\
\hline $\begin{array}{c}\text { Groenewegenand et } \\
\text { al., } 1984^{21} \\
\text { Holanda }\end{array}$ & $\begin{array}{l}\text { Estudo transversal } \\
\text { Observação }\end{array}$ & $\begin{array}{l}\text { Distribuição de } \\
\text { frequência } \\
\text { Híbrido }\end{array}$ & $\begin{array}{l}\text { Independentes da colaboração do } \\
\text { paciente, não provenientes de auto } \\
\text { avaliações, inespecíficos a grupo } \\
\text { populacional e demanda. }\end{array}$ \\
\hline $\begin{array}{l}\text { Thomas-Weintraub, } \\
1985^{22} \\
\text { Estados Unidos da } \\
\text { América }\end{array}$ & $\begin{array}{c}\text { Estudo transversal } \\
\text { Questionário }\end{array}$ & $\begin{array}{l}\text { Distribuição de } \\
\text { frequência } \\
\text { Híbrido }\end{array}$ & $\begin{array}{l}\text { Dependentes da colaboração do } \\
\text { paciente, provenientes de auto } \\
\text { avaliações, específicos a grupo } \\
\text { populacional e inespecíficos a } \\
\text { demanda. }\end{array}$ \\
\hline Strayer et al., $1988^{23}$ & Estudo transversal & Regressão linear & $\begin{array}{l}\text { Dependentes da colaboração do } \\
\text { paciente, provenientes de auto }\end{array}$ \\
\hline
\end{tabular}




\begin{tabular}{|c|c|c|c|}
\hline $\begin{array}{c}\text { Estados Unidos da } \\
\text { América }\end{array}$ & Questionário & Híbrido & $\begin{array}{c}\text { avaliações, específicos a grupo } \\
\text { populacional e inespecíficos a } \\
\text { demanda. }\end{array}$ \\
\hline $\begin{array}{l}\text { Hayward et al., } 198924 \\
\text { Estados Unidos da } \\
\text { América }\end{array}$ & $\begin{array}{c}\text { Estudo transversal } \\
\text { Questionário }\end{array}$ & $\begin{array}{l}\text { Regressão logística } \\
\text { Híbrido }\end{array}$ & $\begin{array}{l}\text { Dependentes da colaboração do } \\
\text { paciente, provenientes de auto } \\
\text { avaliações, específicos a grupo } \\
\text { populacional e inespecíficos a } \\
\text { demanda. }\end{array}$ \\
\hline $\begin{array}{l}\text { Holtzman et al. } 199025 \\
\text { Estados Unidos da } \\
\text { América }\end{array}$ & $\begin{array}{c}\text { Estudo transversal } \\
\text { Questionário }\end{array}$ & $\begin{array}{l}\text { Análise discriminante } \\
\text { Híbrido }\end{array}$ & $\begin{array}{c}\text { Dependentes da colaboração do } \\
\text { paciente, provenientes de auto } \\
\text { avaliações, inespecíficos a grupo } \\
\text { populacional e demanda. }\end{array}$ \\
\hline $\begin{array}{c}\text { Spencer et al., } 1995^{26} \\
\text { Austrália }\end{array}$ & $\begin{array}{c}\text { Estudo longitudinal } \\
\text { Questionário }\end{array}$ & $\begin{array}{l}\text { Regressão logística } \\
\text { Setor Público }\end{array}$ & $\begin{array}{l}\text { Dependentes da colaboração do } \\
\text { paciente, provenientes de auto } \\
\text { avaliações, específicos a grupo } \\
\text { populacional e demanda. }\end{array}$ \\
\hline $\begin{array}{l}\text { Schwarz, } 1996^{27} \\
\text { Dinamarca }\end{array}$ & $\begin{array}{l}\text { Estudo transversal } \\
\text { Questionário }\end{array}$ & $\begin{array}{l}\text { Regressão logística } \\
\text { Híbrido }\end{array}$ & $\begin{array}{l}\text { Dependentes da colaboração do } \\
\text { paciente, provenientes de auto } \\
\text { avaliações, específicos a grupo } \\
\text { populacional e inespecíficos a } \\
\text { demanda. }\end{array}$ \\
\hline $\begin{array}{l}\text { Schwarz, } 1996 a^{28} \\
\text { Dinamarca }\end{array}$ & $\begin{array}{l}\text { Estudo transversal } \\
\text { Observação }\end{array}$ & $\begin{array}{l}\text { Distribuição de } \\
\text { frequência } \\
\text { Híbrido }\end{array}$ & $\begin{array}{l}\text { Independentes da colaboração do } \\
\text { paciente, não provenientes de auto } \\
\text { avaliações, inespecíficos a grupo } \\
\text { populacional e demanda. }\end{array}$ \\
\hline $\begin{array}{l}\text { Honkala et al., } 1996^{29} \\
\text { Finlândia }\end{array}$ & $\begin{array}{c}\text { Estudo transversal } \\
\text { Questionário }\end{array}$ & $\begin{array}{c}\text { Regressão logística } \\
\text { Híbrido }\end{array}$ & $\begin{array}{l}\text { Dependentes da colaboração do } \\
\text { paciente, provenientes de auto } \\
\text { avaliações, específicos a grupo } \\
\text { populacional e inespecíficos a } \\
\text { demanda. }\end{array}$ \\
\hline $\begin{array}{l}\text { Wong et al., } 199730 \\
\text { Inglaterra }\end{array}$ & $\begin{array}{c}\text { Estudo transversal } \\
\text { Observação }\end{array}$ & $\begin{array}{l}\text { Distribuição de } \\
\text { frequência } \\
\text { Setor privado }\end{array}$ & $\begin{array}{l}\text { Independentes da colaboração do } \\
\text { paciente, não provenientes de auto } \\
\text { avaliações, específicos a grupo } \\
\text { populacional e demanda. }\end{array}$ \\
\hline $\begin{array}{l}\text { Porter et al., } 199931 \\
\text { Canadá }\end{array}$ & $\begin{array}{l}\text { Estudo transversal } \\
\text { Observação }\end{array}$ & $\begin{array}{l}\text { Regressão logística } \\
\text { Setor privado }\end{array}$ & $\begin{array}{c}\text { Independentes da colaboração do } \\
\text { paciente, não provenientes de auto } \\
\text { avaliações, inespecíficos a grupo } \\
\text { populacional e direcionados a } \\
\text { demanda específica. }\end{array}$ \\
\hline $\begin{array}{c}\text { Grytten et al., } 2002^{2} \\
\text { Noruega }\end{array}$ & $\begin{array}{c}\text { Estudo transversal } \\
\text { Observação }\end{array}$ & $\begin{array}{c}\text { Regressão logística } \\
\text { Setor privado }\end{array}$ & $\begin{array}{l}\text { Dependentes da colaboração do } \\
\text { paciente, não provenientes de auto } \\
\text { avaliações, específicos a grupo } \\
\text { populacional e inespecíficos a } \\
\text { demanda. }\end{array}$ \\
\hline $\begin{array}{l}\text { Beazoglou et al., } \\
\qquad 2002^{32} \\
\text { Estados Unidos da } \\
\text { América }\end{array}$ & Observação & $\begin{array}{l}\text { Regressão logística } \\
\text { Setor privado }\end{array}$ & $\begin{array}{l}\text { Independentes da colaboração do } \\
\text { paciente, não provenientes de auto } \\
\text { avaliações, inespecíficos a grupo } \\
\text { populacional e demanda. }\end{array}$ \\
\hline
\end{tabular}




\begin{tabular}{|c|c|c|c|}
\hline $\begin{array}{l}\text { Manski et al., } 200433 \\
\text { Estados Unidos da } \\
\text { América }\end{array}$ & $\begin{array}{l}\text { Estudo transversal } \\
\text { Observação }\end{array}$ & $\begin{array}{c}\text { Regressão logística } \\
\text { Híbrido }\end{array}$ & $\begin{array}{l}\text { Dependentes da colaboração do } \\
\text { paciente, provenientes de auto } \\
\text { avaliações, específicos a grupo } \\
\text { populacional e inespecíficos a } \\
\text { demanda. }\end{array}$ \\
\hline $\begin{array}{l}\text { Nietert et al., } 200534 \\
\text { Estados Unidos da } \\
\text { América }\end{array}$ & $\begin{array}{c}\text { Estudo transversal } \\
\text { Observação }\end{array}$ & $\begin{array}{l}\text { Regressão logística } \\
\text { Setor público }\end{array}$ & $\begin{array}{c}\text { Independentes da colaboração do } \\
\text { paciente, não provenientes de auto } \\
\text { avaliações, específicos a grupo } \\
\text { populacional e inespecíficos a } \\
\text { demanda. }\end{array}$ \\
\hline $\begin{array}{c}\text { Matos et al., } 200735 \\
\text { Brasil }\end{array}$ & $\begin{array}{c}\text { Estudo transversal } \\
\text { Questionário }\end{array}$ & $\begin{array}{l}\text { Regressão logística } \\
\text { Híbrido }\end{array}$ & $\begin{array}{l}\text { Dependentes da colaboração do } \\
\text { paciente, provenientes de auto } \\
\text { avaliações, específicos a grupo } \\
\text { populacional e inespecíficos a } \\
\text { demanda. }\end{array}$ \\
\hline $\begin{array}{l}\text { Kleinman et al., } \\
200936 \\
\text { Inglaterra }\end{array}$ & $\begin{array}{c}\text { Estudo transversal } \\
\text { Observação }\end{array}$ & $\begin{array}{l}\text { Distribuição de } \\
\text { frequência } \\
\text { Híbrido }\end{array}$ & $\begin{array}{l}\text { Independentes da colaboração do } \\
\text { paciente, não provenientes de auto } \\
\text { avaliações, in específicos a grupo } \\
\text { populacional e a demanda. }\end{array}$ \\
\hline $\begin{array}{l}\text { Muirhead et al., } \\
200937 \\
\text { Canadá }\end{array}$ & $\begin{array}{c}\text { Estudo transversal } \\
\text { Questionário }\end{array}$ & $\begin{array}{c}\text { Regressão logística } \\
\text { Híbrido }\end{array}$ & $\begin{array}{l}\text { Dependentes da colaboração do } \\
\text { paciente, provenientes de auto } \\
\text { avaliações, específicos a grupo } \\
\text { populacional e inespecíficos a } \\
\text { demanda. }\end{array}$ \\
\hline $\begin{array}{l}\text { Luzzi et al. } 200938 \\
\text { Austrália }\end{array}$ & $\begin{array}{c}\text { Estudo transversal } \\
\text { Questionário }\end{array}$ & $\begin{array}{l}\text { Regressão logística } \\
\text { Setor público }\end{array}$ & $\begin{array}{l}\text { Dependentes da colaboração do } \\
\text { paciente, provenientes de auto } \\
\text { avaliações, específicos a grupo } \\
\text { populacional e demanda. }\end{array}$ \\
\hline $\begin{array}{l}\text { Hicks et al., } 2012^{39} \\
\text { Estados Unidos da } \\
\text { América }\end{array}$ & $\begin{array}{c}\text { Estudo transversal } \\
\text { Questionário }\end{array}$ & $\begin{array}{l}\text { Distribuição de } \\
\text { frequência } \\
\text { Setor privado }\end{array}$ & $\begin{array}{l}\text { Dependentes da colaboração do } \\
\text { paciente, provenientes de auto } \\
\text { avaliações, específicos a grupo } \\
\text { populacional e demanda. }\end{array}$ \\
\hline $\begin{array}{l}\text { Romaire et al., } 2012^{40} \\
\text { Estados Unidos da } \\
\text { América }\end{array}$ & $\begin{array}{l}\text { Estudo transversal } \\
\text { Questionário }\end{array}$ & $\begin{array}{c}\text { Regressão logística } \\
\text { Híbrido }\end{array}$ & $\begin{array}{l}\text { Dependentes da colaboração do } \\
\text { paciente, provenientes de auto } \\
\text { avaliações, específicos a grupo } \\
\text { populacional e inespecíficos a } \\
\text { demanda. }\end{array}$ \\
\hline $\begin{array}{l}\text { Lin et al., } 2012^{41} \\
\text { Taiwan }\end{array}$ & Observação & $\begin{array}{l}\text { Regressão linear } \\
\text { Híbrido }\end{array}$ & $\begin{array}{c}\text { Independentes da colaboração do } \\
\text { paciente, não provenientes de auto } \\
\text { avaliações, inespecíficos a grupo } \\
\text { populacional e demanda. }\end{array}$ \\
\hline $\begin{array}{l}\text { Isong et al., } 201242 \\
\text { Estados Unidos da } \\
\text { América }\end{array}$ & Questionário & Regressão logística & $\begin{array}{l}\text { Dependentes da colaboração do } \\
\text { paciente, provenientes de auto } \\
\text { avaliações, específicos a grupo } \\
\text { populacional e inespecíficos a } \\
\text { demanda. }\end{array}$ \\
\hline Badran et al., $2013^{43}$ & $\begin{array}{c}\text { Estudo transversal } \\
\text { Questionário }\end{array}$ & Regressão logística & $\begin{array}{l}\text { Dependentes da colaboração do } \\
\text { paciente, não provenientes de auto } \\
\text { avaliações, específicos a grupo } \\
\text { populacional e demanda. }\end{array}$ \\
\hline
\end{tabular}




\begin{tabular}{|c|c|c|c|}
\hline $\begin{array}{l}\text { Al-Haboubi et al., } \\
2013^{44} \\
\text { Inglaterra }\end{array}$ & Questionário & Regressão de Poisson & $\begin{array}{l}\text { Dependentes da colaboração do } \\
\text { paciente, provenientes de auto } \\
\text { avaliações, específicos a grupo } \\
\text { populacional e inespecíficos a } \\
\text { demanda. }\end{array}$ \\
\hline $\begin{array}{l}\text { Pinilla et al., } 2015^{45} \\
\text { Espanha }\end{array}$ & Observação & Setor público & $\begin{array}{l}\text { Independentes da colaboração do } \\
\text { paciente, não provenientes de auto } \\
\text { avaliações, específicos a grupos } \\
\text { populacionais e demanda. }\end{array}$ \\
\hline $\begin{array}{l}\text { Schwendicke et al., } \\
\qquad 2016^{46} \\
\text { Alemanha }\end{array}$ & Observação & $\begin{array}{l}\text { Teste de Wilcoxon e } \\
\text { geoprocessamento } \\
\text { Setor público }\end{array}$ & $\begin{array}{l}\text { Independentes da colaboração do } \\
\text { paciente, não provenientes de auto } \\
\text { avaliações, inespecíficos a grupo } \\
\text { populacional e demanda. }\end{array}$ \\
\hline $\begin{array}{l}\text { Jäger et al., } 2016^{47} \\
\text { Alemanha }\end{array}$ & Observação & $\begin{array}{l}\text { Análise de correlação e } \\
\text { geoprocessamento } \\
\text { Setor público }\end{array}$ & $\begin{array}{l}\text { Independentes da colaboração do } \\
\text { paciente, não provenientes de auto } \\
\text { avaliações, inespecíficos a grupo } \\
\text { populacional e demanda. }\end{array}$ \\
\hline $\begin{array}{l}\text { Doan et al., } 2016^{48} \\
\text { Estados Unidos da } \\
\text { América }\end{array}$ & Estudo transversal & $\begin{array}{l}\text { Teste qui-quadrado e } \\
\text { geoprocessamento } \\
\text { Setor público }\end{array}$ & $\begin{array}{l}\text { Independentes da colaboração do } \\
\text { paciente, não provenientes de auto } \\
\text { avaliações, inespecíficos a grupo } \\
\text { populacional e demanda. }\end{array}$ \\
\hline $\begin{array}{l}\text { Tomar et al., } 2016^{49} \\
\text { Estados Unidos da } \\
\text { América }\end{array}$ & Estudo transversal & $\begin{array}{l}\text { Distribuição de } \\
\text { frequência } \\
\text { Híbrido }\end{array}$ & $\begin{array}{l}\text { Independentes da colaboração do } \\
\text { paciente, não provenientes de auto } \\
\text { avaliações, inespecíficos a grupo } \\
\text { populacional e demanda. }\end{array}$ \\
\hline Pilotto et al., 201710 & Estudo transversal & $\begin{array}{c}\text { Regressão logística } \\
\text { Híbrido }\end{array}$ & $\begin{array}{l}\text { Dependentes da colaboração do } \\
\text { paciente, provenientes de auto } \\
\text { avaliações, específicos a grupo } \\
\text { populacional e inespecíficos a } \\
\text { demanda. }\end{array}$ \\
\hline $\begin{array}{l}\text { Singhal et al., } 201750 \\
\text { Canadá }\end{array}$ & Estudo transversal & $\begin{array}{c}\text { Análise de correlação } \\
\text { Setor público }\end{array}$ & $\begin{array}{c}\text { Independentes da colaboração do } \\
\text { paciente, não provenientes de auto } \\
\text { avaliações, não específicos a grupos } \\
\text { populacionais e específicos a } \\
\text { demanda. }\end{array}$ \\
\hline $\begin{array}{l}\text { Leinsalu et al., } 2018^{5} \\
\text { Estônia e Lituânia }\end{array}$ & Estudo transversal & Híbrido & $\begin{array}{l}\text { Dependentes da colaboração do } \\
\text { paciente, provenientes de auto } \\
\text { avaliações, específicos a grupo } \\
\text { populacional e inespecíficos a } \\
\text { demanda. }\end{array}$ \\
\hline $\begin{array}{l}\text { Estados Unidos da } \\
\text { América }\end{array}$ & Questionário & $\begin{array}{l}\text { Regressão linear e } \\
\text { regressão logística } \\
\text { Híbrido }\end{array}$ & $\begin{array}{c}\text { Dependentes da colaboração do } \\
\text { paciente, provenientes de auto } \\
\text { avaliações, específicos a grupo } \\
\text { populacional e demanda. }\end{array}$ \\
\hline
\end{tabular}




\section{Discussão}

Nessa revisão, a temporalidade da maior parte dos estudos foi do tipo transversal2, 5, 10, 22-37, 39-46, 48-50. Apesar da concordância na metodologia empregada, os estudos divergiram em relação à natureza dos dados empregados nas análises. A maior parte dessas avaliações foram embasadas em informações provenientes da aplicação de questionários dirigidos aos pacientes 2 , 10, 23-25, 28, 29, 33, 35, 37, 42, 44, 51 . Entretanto, a aplicação de questionários pressupõe alguma imprecisão nas respostas fornecidas, tanto pela maneira como o entrevistado entende a questão quanto pela forma como a pergunta é construída. 52

Ainda em relação à temporalidade, somente um dos estudos foi do tipo

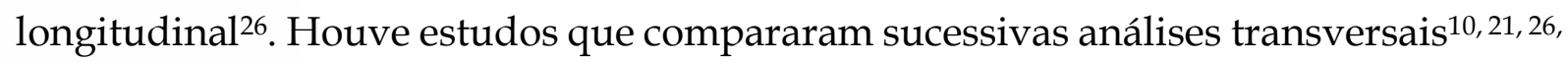
$26,30,34,35,40,42,46,48,51$, um estudo foi descrito como longitudinal do tipo antes e depois 36 e outro estudo descrito como longitudinal ${ }^{45}$, mas após a leitura completa dos textos foi constatado tratar-se de séries de estudos transversais. Portanto, há, nessa revisão, carência de estudos capazes de atribuir fatores causais à demanda por serviços odontológicos. Além da causa, a dissociação da utilização em preventiva e curativa é um dado importante nas avaliações em saúde ${ }^{53}$ que não foi considerado em grande parte dos estudos.

Divergências foram verificadas na caracterização do indivíduo considerado usuário de serviços odontológicos. Algumas análises foram embasadas na resposta referente à realização ou não de consulta ao cirurgião-dentista dentro dos últimos doze meses dentro de um questionário que englobava assuntos diversos², 10, 35, 42, 44. Alguns estudos quantificaram as consultas odontológicas realizadas no ano que antecedeu o estudo 23,33 , ou nos últimos cinco anos 28 , outros exploraram a natureza da consulta odontológica ocorrida ${ }^{24}$ ou investigaram a saúde bucal do entrevistado ${ }^{27}$ e adicionaram à essas informações dados referentes à forma de pagamento pelos erviços ${ }^{44}$. Houve estudos que, apesar de pesquisarem informações sobre o status ental do indivíduo, classificaram como usuário aquele que consultou o cirurgiãontista nos últimos doze meses ${ }^{25}$. Um estudo também julgou usuário o respondente afirmou ter ido ao consultório odontológico nos últimos 24 meses $^{29}$. A ocorrência 
dessas incompatibilidades revelou uma imprecisão no tocante ao delineamento dos estudos.

No que diz respeito ao modelo de análise dos dados, a regressão logística foi o método mais usual. A regressão logística é um método estatístico que testa a interação entre variáveis independentes e seus desfechos. O segundo modelo mais utilizado foi a distribuição de frequências, que compreende a organização dos dados de acordo com as ocorrências dos diferentes resultados observados ${ }^{54}$, e, sendo assim, não objetiva explorar associações entre variáveis.

Apesar da legitimidade de ambos os modelos, verificou-se que os métodos estatísticos fornecem resultados cuja interpretação é complexa nos casos em que mais de duas variáveis são consideradas simultaneamente ${ }^{55}$. Constatação relevante ao considerar a dificuldade de compreensão das avaliações da demanda em saúde decorrente da diversidade de determinantes individuais que ocorrem simultaneamente e cuja segregação é impraticável ${ }^{12}$. Dificuldade esta que é agravada pela relação de dependência demonstrada entre o comportamento da demanda por serviços de saúde e as particularidades que envolvem a assistência em saúde 7,56 . Por isso, há um crescente interesse no uso de técnicas oriundas da inteligência artificial, 57, 58 ainda que nenhum estudo incluído nessa revisão tenha empregado técnicas dessa natureza.

As características extraídas para categorização dos resultados relatados buscaram verificar a reprodutibilidade dos mesmos em cenários diversos. Os resultados descritos foram classificados, primeiramente, como dependentes da colaboração do paciente nos casos em que a ocorrência do estudo dependia da concordância por parte do paciente em algum momento do processo da coleta de dados. Essa característica foi considerada um impedimento da generalização dos desfechos já que os fatores responsáveis pelas diferenças nos níveis de saúde bucal entre as populações envolvem o tipo e a frequência de ingestão alimentar, a prática e frequência de cuidados de higiene oral, o tipo de serviços de saúde, o nível de acesso serviços públicos, as condições socioeconômicas dos indivíduos, as crenças, itos culturais e valores ${ }^{59}$. Verificou-se, assim, que é desejável que as avaliações da 
demanda por serviços odontológicos dissociem os setores público e privado com o intuito de ponderar as disparidades intrínsecas a cada um dos contextos.

Como as características do sistema de saúde local são importantes no processo de investigação da utilização de serviços odontológicos ${ }^{60}$, os desfechos alcançados pelas avaliações incluídas nessa revisão estão sujeitos a vieses inerentes ao seu país de origem. Logo, é relevante o desenvolvimento de estudos nacionais acerca do tema.

Os resultados foram, ainda, classificados como provenientes de auto avaliações uma vez que as limitações referentes ao empregado dessas conjecturas são inevitáveis. No Brasil, a idade foi considerada um fator importante na determinação da validade e da utilidade da auto percepção ${ }^{61}$ e constatou-se que instrumentos válidos em determinados contextos não necessariamente o serão em outros ${ }^{62}$. Ainda no contexto brasileiro, a auto avaliação negativa da saúde bucal foi significativamente maior entre os que apresentaram menor renda e menor escolaridade, e ter realizado a última consulta em consultório público aumentou em 10,0\% a prevalência de auto avaliação negativa da saúde bucal ${ }^{63}$. Essa constatação denota grande peso cultural no teor das auto avaliações.

A limitação dessa revisão foi a inclusão de estudos que analisaram um grupo populacional ou demanda específica. Tais estudos não produziram conhecimento acerca da previsão da demanda por serviços odontológicos em geral, objeto desta revisão, e, mesmo assim, atenderam aos critérios de inclusão desta, denotando falha na delimitação dos critérios de inclusão. Esses textos podem trazer estratégicas eficazes para certos nichos da população ou áreas da Odontologia e, sendo assim, requerem uma avaliação individualizada e aprofundada que foge do escopo desse estudo.

\section{Tonclusões}

A independência da colaboração do paciente, o consenso na literatura da finição do perfil do usuário, o emprego de modelos de análise adequados, o envolvimento de estudos longitudinais, a dissociação dos tratamentos efetivados 
em curativos e preventivos e o direcionamento das análises ao setor público ou privado foram considerados fatores desejáveis para a assertividade e reprodutibilidade das metodologias de interesse dessa revisão.

Os estudos transversais desenvolvidos a partir do emprego de questionários dirigidos aos pacientes, instrumento mais usual, apesar de relevantes, foram considerados limitados para propiciar uma melhor gestão dos recursos e embasar o planejamento de políticas que buscam equilibrar a relação entre a oferta e a demanda por serviços odontológicos. Tal constatação advém do fato de que os resultados desses instrumentos são dependentes da colaboração do paciente e apresentam alto grau de subjetividade.

Contudo, as metodologias empregadas na literatura para prever a demanda por serviços odontológicos foram identificadas. A partir dos resultados dessa revisão, foi gerado conhecimento capaz de apoiar o delineamento de estudos com potencial para aprimorar a previsibilidade da demanda por serviços odontológicos.

\section{Referências}

1. Cameron AC, Trivedi PK, Milne F, Piggot, J. A Microeconometric Model of the Demand for Health Care and Health Insurance in Australia. Review of Economic Studies. 1988 Jan;55(1):85-106.

2. Grytten J Holst, D. Do Young Adults Demand More Dental Services As Their Income Increases? Community Dent Oral Epidemiol. 2002;30(6): 463-9.

3. White $A B$, Factors Influencing Demand for Dental Services: Population, Demographics, Disease, Insurance. J Dent Educ. 2012 Aug;76(8):996-1007.

4. Pinto RS, Matos DL, Loyola Filho AI. Características associadas ao uso de serviços odontológicos públicos pela população adulta brasileira. Ciência e Saúde Coletiva 2012, 17(2):531-544.

Leinsalu M, Reile R, Vals K, et al. Macroeconomic changes and trends in dental care tilization in Estonia and Lithuania in 2004-2012: a repeated cross-sectional study. IC Oral Health. 2018;18(1): 199. doi: 10.1186/s12903-018-0665-5. PubMed PMID: 09245; PubMed Central PMCID: PMC6276228.

ôte MJ, Tucker SL. Four methodologies to improve healthcare demand forecasting. 1thc Financ Manage. 2001 May;55(5):54-8. 
7. Birch, S. Demand-based models and market failure in health care: projecting shortages and surpluses in doctors and nurses. Health Economics, Policy and Law. 2018 Aug; 14(2): 291-294.

8. Ntabaye MK, Scheutz F, Poulsen S. Patient satisfaction with emergency oral health care in rural Tanzania. Community Dent Oral Epidemiol. 1998 Oct; 26(5): 289-95.

9. Manski RJ. Projecting the Demand for Dental Care in 2040. J Dent Educ. 2017 Aug; 81(8) Supplement: eS133-45.

10. Pilotto LM, Celeste RK. Tendências no uso de serviços de saúde médicos e odontológicos e a relação com nível educacional e posse de plano privado de saúde no Brasil, 1998-2013. Cad. Saúde Pública. 2017 Mar;34(4): e00052017.

11. Conrad D, Lee SYR, Milgrom P, et al. Estimating determinants of Dentist productivity: new Evidence. J public Health Dent. 2010;70(4):262-8.

12. Guay A, Blatz A. The effect of the Great Recession on the demand for general oral health care and orthodontic care. The Journal of the American Dental Association. 2019 Abr;150(4): 287-93.

13. Pierantoni CR, França T, Magnago C, et al. Graduações em Saúde no Brasil: 2000 2010. 1st ed. Rio de Janeiro: Centro de Estudos e Pesquisa em Saúde Coletiva CEPESC, 2012.

14. http:// www.ans.gov.br [internet]. [Rio de Janeiro, RJ]: Agência Nacional de Saúde Suplementar - ANS; c2019 [Acesso em 2019 Abr 28]. Disponível em: http://www.ans.gov.br/sala-de-imprensa/releases/consumidor/3531-ans-propoemelhoria-do-cuidado-odontologico-prestado-pelos-planos-de-saude.

15. https://www.iess.org.br/cms/rep/NAB\%2043.pdf. [internet]: Instituto de Estudos de Saúde Suplementar - IESS; Nota de Acompanhamento de Beneficiários: NAB: Edição 42. c2020. [Acesso em 2020 Mai 17] Disponível em: https://www.iess.org.br/cms/rep/NAB\%2043.pdf.

16. https:/ / www.census.gov [internet]. Suitland: United States Census Bureau; c2019 [Acesso em 2019 Abr 14]. Disponível em: https://www.census.gov/popclock/.

17. https://datausa.io. [internet]. Data USA; c2019 [Acesso em 2019 Abr 14]. isponível em: https://datausa.io/profile/soc/dentists.

Botelho L.L.R., Cunha C.C.A., Macedo M. O método da revisão integrativa nos tudos organizacionais. Gestão e Sociedade. 2011 Mai-Ago;5(11):121-36. 
19. Moher D, Liberati A, Tetzlaff J, et al. The PRISMA Group. Preferred Reporting Items for Systematic Reviews and Meta-Analyses: The PRISMA Statement. PLoS Med. 2009;6(7): e1000097.

20. Whittemore R, Knafl K. The integrative review: updated methodology. Journal of Advanced Nursing. 2005;52(5):546-53.

21. Groenewegenand PP, Postma JHM. The supply and utilization of dental services. Social Science and Medicine. 1984;19(4):451-59.

22. Thomas-Weintraub A. Dental needs and dental service use patterns of an elderly edentulous population. The Journal of Prosthetic Dentistry. 1985 Out;54(4): 526-32.

23. Strayer MS, Branch LG, Jones JA, et al. Predictors of the use of dental services by older veterans. Special Care in Dentistry. 1988;8(5):209-13.

24. Hayward RA, Meetz HK, Shapiro MF, et al. Utilization of dental services: 1986 patterns and trends. Journal of Public Health Dentistry. 1989;49(3):147-52.

25. Holtzman JM, Berkey AB, Mann J. Predicting Utilization of Dental Services by the Aged. Journal of Public Health Dentistry.1990;50(3):164-71.

26. Spencer AJ, Allister JH, Brennan DS. Predictors of fixed orthodontic treatment in 15-year-old adolescents in South Australia. Community Dent Oral Epidemiol. 1995;23(6):350-55.

27. Schwarz E. Changes in utilization and cost sharing within the Danish National Health Insurance dental program, 1975-90. Acta Odontologica Scandinavica. 1996;54(1):29-35.

28. Schwarz E. Changes in demand for dental care among Danish adults, 1975-90. Acta Odontologica Scandinavica. 1996a;54(1):36-43.

29. Honkala E, Kuusela S, Rimpelä A, et al. Dental services utilization between 1977 and 1995 by Finnish adolescents of different socioeconomic levels. Community Dent Oral Epidemiol. 1997;25(6):385-90.

30. Wong FS, Fearne JM, Brook AH. Planning future general anaesthetic services in paediatric dentistry on the basis of evidence: An analysis of children treated in the Day Stay Centre at the Royal Hospitals NHS Trust, London, between 1985-95. International Dental Journal, 1997;47(5):285-92.

Porter J, Coyte PC, Barnsley J, et al. The effects of fee bundling on dental utilization. alth Services Research. 1999 Out;34(4): 901-21. 
32. Beazoglou, T, Bailit H, Heffley D. The dental work force in Wisconsin: Ten-year projections. The Journal of the American Dental Association. 2002 Ago;133(8):10981104.

33. Manski RJ, Goodman HS, Reid BC, et al. Dental Insurance Visits and Expenditures among Older Adults. American Journal of Public Health. 2004 Mai;94(5):759-64.

34. Nietert PJ, Bradford WD, Kaste LM. The impact of an innovative reform to the South Carolina Dental Medicaid System. Health Services Research, 2005 Ago;40(4): 1078-91, Aug.

35. Matos DL, Lima-Costa MF. Trends in the use of dental services by elderly Brazilians and related socio-demographic factors based on the National Household Survey (1998 and 2003). Cad. Saúde Pública. 2007 Nov;23(11):2740-48.

36. Kleinman ER, Harper PR, Gallagher JE. Trends in NHS primary dental care for older people in England: Implications for the future. Gerodontology. 2009;26(3):193201.

37. Muirhead VE, Quiñonez C, Figueiredo R, et al. Predictors of dental care utilization among working poor Canadians. Community Dent Oral Epidemiol. 2009;37(3):199208.

38. Luzzi L, Jones K, Spencer AJ, et al. Association of urgent dental care with subjective oral health indicators and psychosocial impact. Community Dental Health Journal. $2009 ; 26(2) 77-83$.

39. Hicks CG, Jones JE, Saxen MA, et al. Demand in pediatric dentistry for sedation and general anesthesia by dentist anesthesiologists: a survey of directors of dentist anesthesiologist and pediatric dentistry residencies. Anesthesia Progress. 2012;59(1):311.

40. Romaire MA, Bell JF, Huebner CE. Variations in children's dental service use based on four national health surveys. Pediatrics. 2012 Out; 130(5): e1182-89.

41. Lin C, Chao H. Use of selected ambulatory dental services in Taiwan before and after global budgeting: a longitudinal study to identify trends in hospital and clinicbased services. BMC Health Services Research 2012 Set: 12:339.

42. Isong IA, Soobader MJ, Fisher-Owens SA, et al. Racial disparity trends in children's dental visits: US National Health Interview Survey, 1964-2010. Pediatrics, 2012 go;130(2): 306-11.

Badran AS, Al-Khateeb S. Factors influencing the uptake of orthodontic treatment. urnal of Public Health Dentistry. 2013;73(4):339-44. 
44. Al-Haboubi M, Klass C, Jones $\mathrm{K}$, et al. Inequalities in the use of dental services among adults in inner South East London. European Journal of Oral Sciences, 2013;121(3 Pt 1):176-81.

45. Pinilla J, Hernández MAN, Abásolo I. Time trends in socio-economic inequalities in the lack of access to dental services among children in Spain 1987-2011. Int J Equity Health, 2015 Jan;14:9.

46. Schwendicke F, Jäger R, Hoffmann W, et al. Estimating spatially specific demand and supply of dental services: a longitudinal comparison in Northern Germany. Journal of Public Health Dentistry. 2016;76(4):269-75.

47. Jäger R, Van Den Berg N, Hoffmann W, et al. Estimating future dental services' demand and supply: A model for Northern Germany. Community Dent Oral Epidemiol. 2016;44(2):169-79.

48. Doan L, Tiwari T, Brunson D, et al. Medicaid Adult Dental Benefit Impact on Dental Utilization: A University Clinic Setting. Frontiers in Public Health, 2017 Jul;5:147. doi: 10.3389/fpubh.2017.00147. PubMed PMID: 28725642; PubMed Central PMCID: PMC5495854.

49. Tomar SL, Carden DL, Dodd VJ, Catalanotto FA, Herndon JB. Trends in DentalRelated Use of Hospital Emergency Departments in Florida. J Public Health Dent. 2016 June;76(3): 249-257.

50. Singhal S, McLaren L, Quiñonez C. Trends in emergency department visits for nontraumatic dental conditions in Ontario from 2006 to 2014. Can J Public Health 2017;108(3): e246-e250.

51. Luo H, Bell RA, Wright W, et al. Trends in annual dental visits among US dentate adults with and without self-reported diabetes and prediabetes, 2004-2014. The Journal of the American Dental Association. 2018;49(6): 460-69.

52. Nascimento AR, Andrade FB, César CC. Fatores associados à concordância entre autopercepção e avaliação clínica da necessidade de tratamento dentário em adultos do Brasil e de Minas Gerais. Cad. Saúde Pública. 2016 Out;32(10): e00039115.

53. Hyman J. The limitations of using insurance data for research. The Journal of the American Dental Association. 2015 Mai;146(5):283-5.

4. Barbetta, PA. Estatística aplicada às ciências sociais.7th ed. Florianópolis: Editora Universidade Federal de Santa Catarina - UFSC; 2007.

$\mathrm{Hu} \mathrm{YH}$, Tai CT, Chen SCC, et al. Predicting return visits to the emergency partment for pediatric patients: Applying supervised learning techniques to the 
Taiwan National Health Insurance Research Database. Comput Methods Programs Biomed; 2017 Jun; 144: 105-112.

56. Barros, PP; Machado, MP.; Sanz-de-Galdeano, A. Moral hazard and the demand for health services: A matching estimator approach. Journal of Health Economics; 2008 Jul; 27(4): 1006-1025.

57. Toti G, Vilalta R, Lindner P, et al. Analysis of correlation between pediatric asthma exacerbation and exposure to pollutant mixtures with association rule mining. Artificial Intelligence in Medicine. 2016 Nov;74(1):44-52.

58. Ariyadasa SN, Munasinghe KL, Senanayake HDS, et al. Knowledge Extraction to Mitigate Child Malnutrition in Developing Countries (Sri Lankan Context). Conference presented at: 2013 4th International Conference on Intelligent Systems, Modelling and Simulation; 2013 Jan 29-31, Bangkok, Thailand. doi:10.1109/ISMS.2013.23.

59. Reis LM, Ramos N, Migração, Aculturação e Saúde Bucal de Brasileiras e Brasileiros Residentes em Lisboa, Portugal. Revista Ciência Plural. 2016;2(1):56-68.

60. Dho MS. Factores associados a la utilización de servicios de salud odontológicos. Ciência e Saúde Coletiva. 2015;23(2):509-18.

61. Nascimento AR, Andrade FB, César CC. Validity and utility of self-perceived need for dental treatment by adults and elderly. Cad Saúde Pública. 2015 Ago;31(8):176574 .

62. Ramos RQ, Bastos JL, Peres MA. Validade diagnóstica de agravos bucais autorreferidos em inquéritos populacionais: revisão da literatura. Rev Bras Epidemiol 2013; 16(3): 716-28.

63. Luchi CA, Peres KG, Bastos JL, et al. Desigualdades na autoavaliação da saúde bucal em adultos. Rev Saúde Pública. 2013;47(4):740-51. 